\title{
Association suisse de Médecine de la Musique
}

\author{
L'Association suisse de Médecine de la Musique (SMM) a été fondée en 1997. Cette \\ branche spécifique de la médecine du travail comprend des médecins, des dentistes \\ et des thérapeutes issus de différents domaines. Elle fait office, pour la Suisse entière, \\ de bureau de coordination et de conseil pour la médecine de la musique.
}

Adrian Sury

Correspondance:

Dr Adrian Sury

Médecine générale FMH et médecine d'entreprise SSMT Via della Posta 44

CH-Locarno

adrian.sury@hin.ch

Bureau de coordination et de conseil pour la médecine de la musique

Rumiweg 4

CH-4539 Farnern

info@musik-medizin.ch

www.musik.medizin.ch
Un nombre croissant de musiciens est aujourd'hui confronté à des problèmes spécifiques qui tendent à restreindre leur aptitude au jeu musical et qui les empêchent même de travailler. De nos jours, la musique professionnelle est comparable à un sport de haut niveau exigeant des performances très élevées. Souvent, les traitements symptomatiques ne mènent qu'à une amélioration de courte durée ou aggravent même les troubles. Aussi est-il important d'identifier les causes et de prendre le musicien au sérieux dans toute sa spécificité.

C'est précisément dans ce but que s'engage la Société Suisse pour la Médecine de la Musique (SMM). La SMM collabore avec des institutions et des spécialistes sélectionnés qui ont plusieurs années d'expérience dans le domaine de la médecine de la musique et qui amènent donc des connaissances médicales et musicales. Elle met en avant une collaboration étroite et interdisciplinaire entre médecins, dentistes et thérapeutes, afin que le patient soit perçu en tant que musicien et non en tant que simple patient.

En Suisse, la SMM a joué un rôle de pionnier dans la prévention et le traitement des problèmes de santé des musiciens. Il y a deux décennies, en rédigeant sa thèse sur les «Problèmes prothétiques et le traitement de musiciens d'harmonie» (Prothetische Probleme bei der Behandlung von Blasmusikern), le docteur Markus Keller, dentiste, trompettiste et ancien viceprésident de la SMM, a cherché en vain des spécialistes ou des publications en Europe centrale ou même en Suisse. A l'époque, on trouvait quelques rares médecins pour musiciens en Angleterre et dans l'ex-RDA, où il existait une médecine du travail pour musiciens au sein des services ambulatoires des théâtres berlinois (Berliner Bühne). Dans notre pays, malheureusement, aucune infrastructure de ce genre n'était en place. En 1997, la kinésiologue et tromboniste professionnelle Pia Bucher ainsi que le dentiste et pianiste Joachim Lahme mirent heureusement un terme à cette situation regrettable en créant la SMM. Grâce à eux, la médecine de la musique a trouvé sa place en Suisse. De nombreux cours de formation, conférences, colloques et documents ont permis à la SMM de devenir ce qu'elle est aujourd'hui: une société spécialisée reconnue et ayant des contacts bien au-delà des frontières nationales, une organisa-

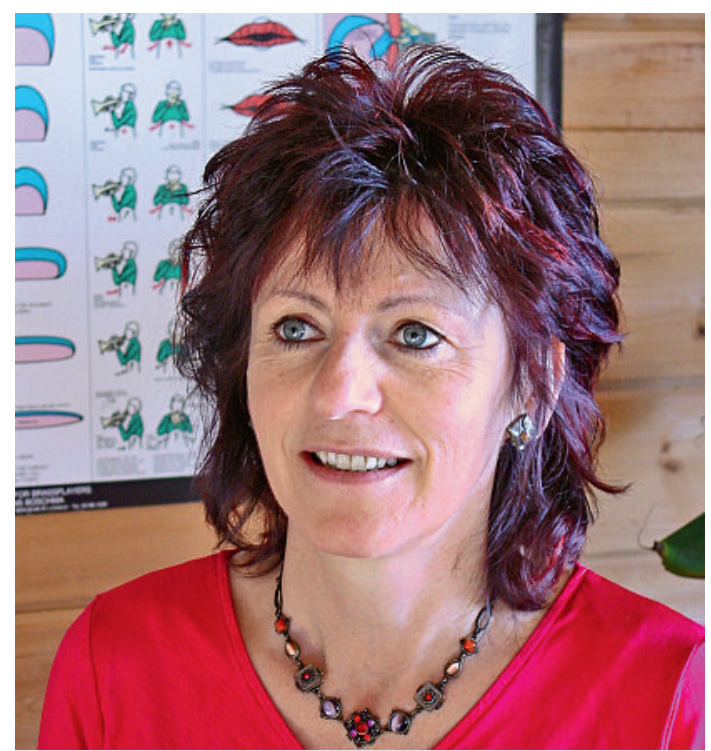

Pour la remercier de l'excellent travail fourni en tant que présidente, Pia Bucher a été nommée présidente honoraire.

tion pour la formation continue et un bureau de coordination pour musiciens, médecins et thérapeutes. La préoccupation première de ces spécialistes est le bien-être médical des artistes et des nombreux musiciens amateurs. La société compte aujourd'hui 117 membres actifs, présents dans toutes les régions du pays et organisés en services de consultation interdisciplinaires pour musiciens.

La fondatrice de la SMM Pia Bucher s'est retirée de ses fonctions de présidente le 22 janvier 2011; Martina Berchtold, psychologue et violoniste, a été choisie pour lui succéder. Le docteur Jürg Frei, médecin interniste et flûtiste, a repris la vice-présidence. Font également partie du comité de direction Fredy Bopp, physiothérapeute et bassiste, et Sylvia Schwarzenbach, pédagogue et clarinettiste. Un service de conseil professionnel composé de médecins, de dentistes et de thérapeutes originaires de toutes les régions du pays complète le comité. Pour la remercier de l'excellent travail fourni en tant que présidente, Pia Bucher a été nommée présidente honoraire.

Le prochain colloque aura lieu le 22 octobre 2011 à l’Université des Arts à Berne. 